\title{
Las citaciones científicas: redes de referencias en universos de referencias. El ejemplo de los artículos de química ${ }^{1}$.
}

\author{
Béatrice Milard² - LISST (Universidad de Toulouse, Francia)
}

\section{Resumen}

El objetivo general de mi investigación es hacer evidente el espesor social y relacional de las referencias y citaciones científicas mostrando que, lejos de ser solamente indicadores de productividad o de visibilidad de la investigación, también son una expresión de la sociabilidad científica. Al ser las que dinamizan las relaciones sociales e intelectuales de los investigadores, las referencias y las citaciones son el posible vector de transformación de los colectivos científicos.

Basándome en un corpus de 32 artículos procedentes de un laboratorio de química de Toulouse y con el apoyo de entrevistas a sus autores, analizo las referencias de tres de estos artículos como una ocasión para sus autores de dinamizar las relaciones con los colectivos a los que se declaran pertenecer, o con los que se confrontan por medio de estas referencias. Continúo mostrando que las redes de referencia de cada publicación se inscriben en el universo de referencia (conjunto de citaciones que son referencia del artículo) que ellas mismas contribuyen en parte a crear.

Palabras clave: ciencia - publicaciones científicas - referencias - redes de referencia - citaciones - cocitaciones - universos de referencia

\begin{abstract}
The overall goal of my research is to highlight the social and relational depth of references and citations in scientific evidence that, far from being mere indicators of productivity and visibility of research, are also an expression of scientific sociability. Because they boost the social and intellectual research, references and citations are the possible vector for transformation of scientific community.

Based on a corpus of 32 articles from a chemistry laboratory in Toulouse and with interviews with their authors, I analyze the references of three of them as an opportunity for authors, to boost their relations with collectives to whom they claim to belong or they oppose through these references. I continue by showing that the networks of references of each publication are embedded in a universe of references (all citations that references of the article have generated in the $\mathrm{SCl}$ Thomson Reuters, before and after its publication) that they partly contribute to create.
\end{abstract}

Key words: science - scientific publications- references - networks of references citations - cocitations - universes of reference

\footnotetext{
${ }^{1}$ Traducción: Sandra Corcuera Fortuny

${ }^{2}$ Contacto : beatrice.milard@univ-tlse2.fr
} 


\section{I ntroducción}

Las referencias y las citaciones científicas rara vez han sido objeto de análisis que tengan en cuenta su contenido relacional. Los trabajos que se interesan en la actividad de investigación por el estudio de la construcción social de los escritos científicos, a menudo ya no prestan atención a los documentos una vez terminados de redactar.

A la inversa, los estudios bibliométricos que se focalizan en la visibilidad de los documentos publicados, soslayan con demasiada frecuencia la dimensión social que acompaña su realización y difusión. Para traspasar estos límites, propongo un estudio de las publicaciones científicas que se centra en las dinámicas relacionales de las referencias y las citaciones ${ }^{3}$.

\section{Dinámicas relacionales de citaciones y referencias científicas}

Las citaciones científicas han sido desde hace mucho tiempo un objeto privilegiado de los analistas de la ciencia y, en primer lugar, de los trabajos relevantes en el campo de la cienciometría. Desde los primeros análisis de Merton y su equipo sobre el «Efecto Mateo » (Merton, 1968) y la crítica de «La hipótesis de Ortega » (Cole y Cole, 1967), se han evidenciado los efectos de contexto (prestigio de la universidad, trayectoria de los investigadores, demografía, repartición estructural de las tasas de citaciones) y sobre todo acumulativos sobre la productividad y la visibilidad (las citaciones) de los investigadores. Sin embargo, estos trabajos no pretenden explicar cómo se suelen producir las jerarquías, cómo se difunden y menos aún cómo participan en éste fenómeno las actividades de referencia. El análisis de los "colegios invisibles" (Price, 1966 y Crane, 1972), es decir, las redes informales relacionadas con los intercambios intelectuales, se atribuyó en cierta forma este objetivo, especialmente con el estudio de las cocitaciones. Así, se ha mostrado el papel de las referencias bibliográficas en la construcción de las "áreas de investigación", así pues, de las especialidades disciplinarias (Small y Griffith, 1974; Small, 1979; Hargens y Felmlee, 1984; Hargens 2000), en su dinámica (Mullins, 1972; Michaelson, 1993) y porosidad (Callon, Law, y Rip, 1986; Callon, Courtial, y Penan, 1993). No obstante, estos estudios sobre los colegios invisibles,

\footnotetext{
${ }^{3}$ Para los cienciómetros, la diferencia entre « citaciones » y « referencias » está claramente establecida desde hace tiempo : si una publicación A menciona en la bibliografía a un documento $B$, el artículo A hace una referencia al artículo B, y el artículo B es una citación del artículo A (Price, 1970). Me acogeré a esta distinción en este texto.
} 
como aquellos sobre la estratificación de la ciencia, a pesar de todas sus aportaciones presentan la común limitación de no considerar la referencia o la citación más que como un indicador que expresa la especialización o el reconocimiento, y no, como yo propongo hacer, como una expresión de la sociabilidad científica y profesional.

Los trabajos de sociología de las ciencias que desarrollan una aproximación más pragmática, abordan las referencias como elementos persuasivos (Gilbert, 1977), aliados a los que hay que interesar y movilizar para construir la credibilidad del texto (Latour, 1977; Callon et al. 1984). El análisis de los discursos de los científicos revela que las referencias tiene diferentes "funciones" (Moravcsik y Murugesan, 1975; Chubin y Moitra, 1975), incluyendo negativas (MacRoberts y MacRoberts, 1984). Se establecieron tipologías y aún ahora nos preguntamos sobre la complejidad de las motivaciones a citar mostrando todas las sutilezas de comunicación que generan, en especial en función de las disciplinas (Brooks, 1985; 1986; Harwood, 2008; 2009). Sin embargo, estos trabajos de estudios de las ciencias se focalizan muy a menudo sobre la dimensión normativa (Cronin, 1984) o retórica de la referencia y tampoco prestan jamás verdadera atención a su contenido relacional (en particular porque cuando se interesan en las referencias, los trabajos aportados sobrepasan rara vez el tiempo de la escritura de la publicación). De esta manera, no se sabe nada de las relaciones que unen a los autores a sus referencias, ni a las referencias entre ellas, y tampoco se sabe lo que la publicación "expresa" desde un punto de vista relacional. Por estas razones, es pertinente desarrollar un análisis que se centre en las dinámicas relacionales de las referencias.

Por el lado del análisis de las redes sociales, nos interesamos cada vez más en las publicaciones científicas. Desde hace diez años, ciertos trabajos han mostrado que la estructura social (las relaciones personales) se reflejan por una parte en la estructura cognitiva (las referencias y las citaciones): Baldi, 1998; White, 2001; Cronin y Shaw, 2002; White, Wellman y Nacer, 2004. Actualmente, nos preguntamos sobre las coincidencias de las relaciones sociales (colaboraciones) y cognitivas (cocitaciones) en los grupos de investigadores: por ejemplo, Tuire y Erno, 2001; Lazega et al. 2004; Roth, 2008; Roth y Cointet, 2009. Sin embargo, el carácter formal y la ambición de estos trabajos de ser un modelo, no permiten apoderarse de la dimensión social de los intercambios relacionados con las referencias y las citaciones en sus diferentes formas, su intensidad, su historia. 
Se trata de aportar un esclarecimiento más cualitativo y concreto sobre estas cuestiones mostrando que las referencias citadas no son simplemente un eco de la sociabilidad científica, que tampoco se limitan únicamente a coincidir con ella, sino que son la ocasión de activar ciertos vínculos, de relacionar entre sí a ciertos integrantes de la investigación. Este enfoque permite particularmente comprender mejor cómo los textos contribuyen a determinar, consolidar o destruir las relaciones entre ciertos grupos, y participan en la formación de los colectivos científicos.

El estudio del papel que desempeñan los textos científicos y las citaciones en la estructuración de las relaciones sociales y los colectivos científicos requiere poner en marcha un procedimiento plural, que no se limite a la formalización y a la modelización y que al mismo tiempo no evite recurrir a datos cuantitativos de contenido más macrosociológico. Por esta razón he confeccionado un método "mixto" que asocia procedimientos cualitativos (entrevistas a los investigadores y análisis de contenido de las publicaciones) y cuantitativos (codificación sistemática de algunas de las informaciones recogidas en la entrevista y análisis de contenido y extracción de datos bibliográficos).

Realicé 32 entrevistas a investigadores de un laboratorio de química en Toulouse organizando cada una de ellas entorno a una de sus publicaciones. Utilicé la bibliografía del texto como "generador de nombres" para comprender la red sociocognitiva (intelectual, profesional y social) relacionada con esta publicación. Los investigadores caracterizaron el tipo de relación que tenían con cada persona de la referencia citada y, si no la conocían personalmente, me precisaron lo que sabían de ella. Completé estas informaciones de tipo "red personal" con una descripción detallada del conjunto del proceso de publicación del artículo desde el punto de vista de su autor: escritura, evaluación, cooperaciones, financiamientos, publicaciones y colaboraciones anteriores y posteriores. Finalmente, también recabé otras informaciones utilizando las bases de datos bibliográficas en vigor en la disciplina con el objetivo de reunir datos de orden bibliométrico sobre las referencias estudiadas.

De momento, sólo 15 publicaciones fueron objeto de un análisis avanzado. Los autores entrevistados son investigadores del CNRS*, tan sólo uno de ellos es profesor investigador en la universidad. Presentan una heterogeneidad relativa, ya que once de ellos son hombres y cuatro son mujeres; seis defendieron su tesis en los años setenta o antes, cuatro en los ochenta, y diez en los noventa o en los dos

\footnotetext{
* (N. del T.) CNRS : Centre National de la Recherche Scientifique (Centro Nacional de Investigación Científica), su equivalente en España es el « Consejo Superior de Investigaciones Científicas ».
} 
mil. En la mayoría de los casos, las publicaciones aparecieron en una revista selectiva de la especialidad y el investigador entrevistado es su referente (en química, se coloca un asterisco en uno solo de los firmantes del artículo, el referente, lo que deja claro las cuestiones de responsabilidad). Diez de las publicaciones son artículos cortos y cinco son largos. Las quince publicaciones estudiadas reúnen 634 referencias que incluyen a 2.381 autores. La reseña de las publicaciones que citan las mismas referencias se realizó con la ayuda de la base de datos bibliográficos "Web of Science" (WoS) de Thomson Reuters, que recoge lo esencial de la producción literaria de la disciplina. Entre 1950 y 2010, las 634 referencias del corpus se citan en 131.091 publicaciones registradas por la WoS.

El análisis de la relación de los investigadores entrevistados con los autores citados en referencias en sus artículos, muestra una sociabilidad profesional que presenta características similares a las sociabilidades más ordinarias (Milard, 2010). Incluso si los investigadores entrevistados no conocen a todos los autores que citaron en la bibliografía (sólo un $50 \%$ de los 2.381 autores), ubican fácilmente la presencia de dos equipos o del responsable de la publicación o del jefe del equipo de dicha referencia. Casi no describen sus relaciones en función de sus afiliaciones institucionales (especialmente entre investigadores franceses cuyas afiliaciones son particularmente híbridas, véase Grossetti et Milard, 2003) sino más bien en función de sus trayectorias de investigación, sus especialidades a un nivel bastante general (o la disciplina cuando participan varios) y, finalmente, su origen geográfico. De manera general, he podido notar una tendencia a la homofilia entre los autores que citan y los autores de sus referencias, desde el punto de vista de la disciplina, del origen geográfico o de la afiliación institucional. Asimismo, la naturaleza de las relaciones se parece a la que encontramos en el mundo social en general: existen pocos vínculos muy fuertes (el equipo, los "viejos conocidos", vínculos de amistades...) y muchos vínculos débiles (breves encuentros en congresos, simplemente coincidieron en un mismo lugar) entre los que citan y los citados. Así pues, podemos concluir con que la publicación es un marco pertinente para la expresión de la sociabilidad científica.

Incluso si los vínculos que les sirven de base expresan al final una sociabilidad ordinaria, tengo la hipótesis de que las referencias no son un simple eco, ni un reflejo de la sociabilidad científica, sino también la ocasión de visualizar, señalar, activar ciertos vínculos (cognitivos y sociales). Las referencias son pues la ocasión (implícita o explícita) de determinar, consolidar o destruir las relaciones entre ciertos grupos. Para poner a prueba esta hipótesis y sus implicaciones, debemos observar la literatura científica anterior y posterior al artículo (el "universo de 
referencia"), si existen rastros de estas tentativas de organización de los grupos. Son huellas que expresan el resultado de la publicación desde un punto de vista relacional y sociocognitivo.

Empezaré por la descripción de las "redes de referencia" de tres artículos, muy distintos entre sí; qué colectivos se señalan a través de las referencias de un artículo y la elección de dichas referencias, ¿qué dinámicas revelan? Continuaré con el estudio de los "universos de referencia" de estos tres artículos, es decir, el conjunto de las publicaciones una vez citadas las mismas referencias que el artículo estudiado. ¿Existe un "antes" y un "después" de la publicación desde el punto de vista de la organización de los colectivos cognitivos (de las cocitaciones de estas referencias)?

\section{Redes de referencia y descripción de colectivos}

La "red de referencia" de una publicación es una forma de dar cuenta de la organización de las referencias tal como el investigador ha escogido exponerlas. Consiste en considerar cada referencia como un punto de la red y relacionar las que tengan al menos un autor en común. En realidad, abreviamos con la expresión "red de referencia" lo que deberíamos llamar más precisamente "red de autores en común en las referencias". Esta presentación permite ubicar los grupos convocados y visibilizados en la publicación, y saber en qué medida la red está centrada en una o varias personas en particular. De hecho, citar varias veces a la misma persona (o al mismo equipo) en la publicación le otorga importancia, hasta el punto de conllevar a veces que el editor de la revista lo nombre como experto del artículo. Con la ayuda de las informaciones recogidas en la entrevista, la red de referencia se puede dibujar ${ }^{4}$ y calificar de manera que identifique los eventuales grupos o componentes que pueden surgir.

Las tres publicaciones escogidas aparecieron en revistas muy selectas. Tienen la particularidad de presentar redes de referencia muy diferentes desde el punto de vista de su densidad y su forma: la primera es tricéfala, la segunda tiene múltiples componentes, y la tercera es muy dispersa.

\footnotetext{
${ }^{4}$ Las redes de las figuras 1 a la 6 están diseñadas a mano.
} 


\section{Ejemplo de una red de referencia densa y polarizada}

La primera publicación apareció en Proceedings of The Nacional Academy of Sciences of the USA (PNAS) en 2003. Es un artículo corto de cuatro páginas firmado por cuatro autores, dos en postdoctorado, un investigador, y el jefe del equipo. La persona entrevistada (la que tiene el asterisco) es uno de los estudiantes de post-doctorado, que durante sus estudios, se hizo investigador en Toulouse. El artículo incluye 23 referencias, en al menos una de ellas el autor del artículo conoce al autor de la referencia, y en siete de las referencias, el autor del artículo desconoce a sus autores ${ }^{5}$. Los números de los puntos están en el orden de aparición de las referencias en la publicación.

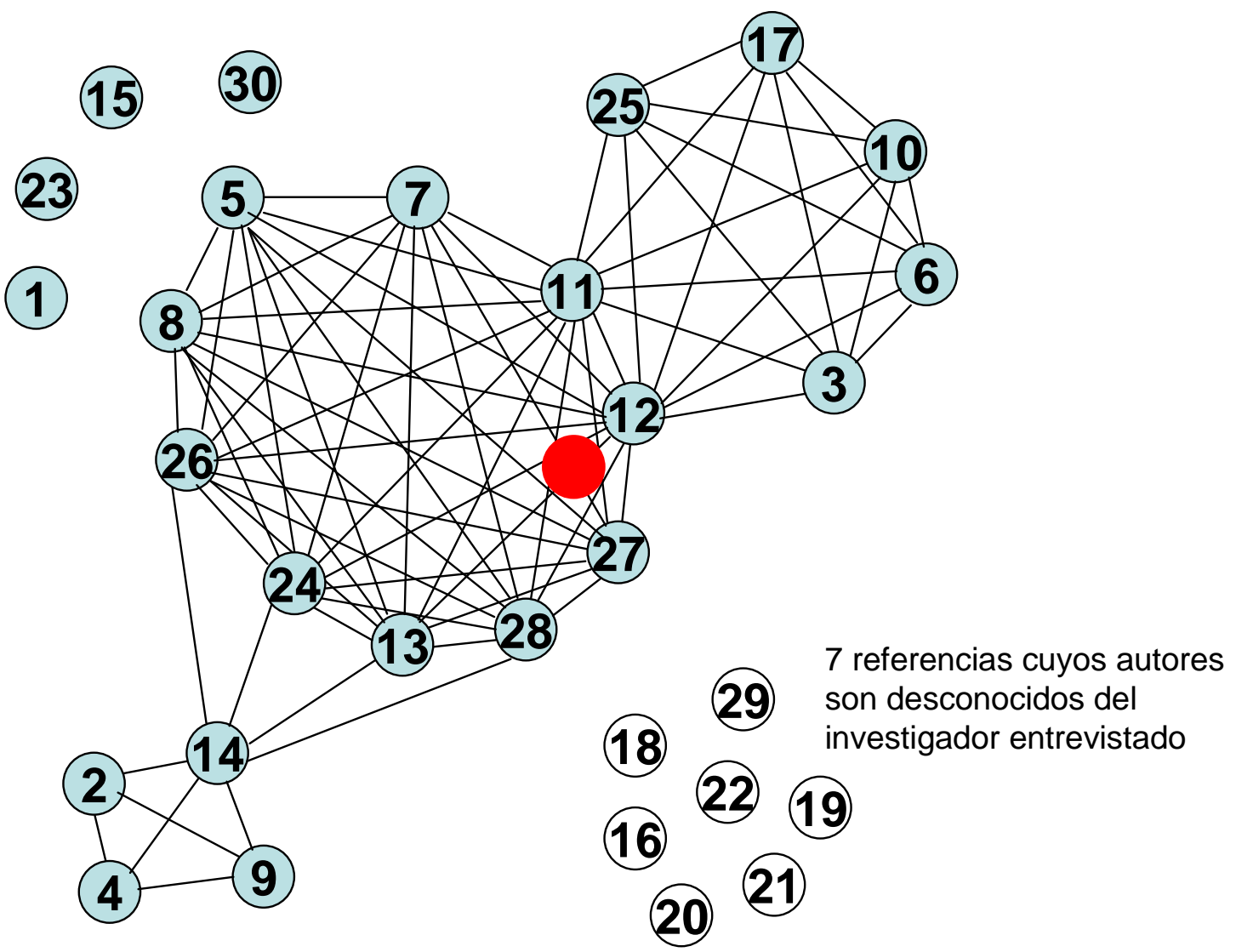

Figura 1. Red de referencias del artículo aparecido en PNAS en 2003

La red de referencia presenta una gran densidad. Tan sólo cuatro referencias (además de las siete cuyos autores no son conocidos por el investigador entrevistado) no están vinculadas con las otras. Observamos una red principal y dos componentes adyacentes relacionados con la red principal por uno o dos artículos. ¿Qué significa esta forma, a qué nos reenvía? Las referencias donde encontramos al investigador entrevistado como autor son las número $7,11,12,27$,

\footnotetext{
${ }^{5}$ Las referencias a otros autores que el investigador entrevistado no conoce, están dibujadas en la Figura 1; en las figuras 3 y 5 sólamente se señalan.
} 
y 28 (ver círculo rojo en las figuras 1 y 2). La publicación de 2003 surgió al hilo de otras dos citadas aquí, la número 11 y la número 12 (en el centro derecho de la figura 1), aparecidas en 2001 y 2002, sobre el mismo tema. La primera consistía en contradecir lo que ya había sido mostrado sobre el tema, y la segunda añadía un nivel suplementario proponiendo predicciones.

La figura 2 a continuación visualiza los grupos tal como han sido descritos por el investigador entrevistado.

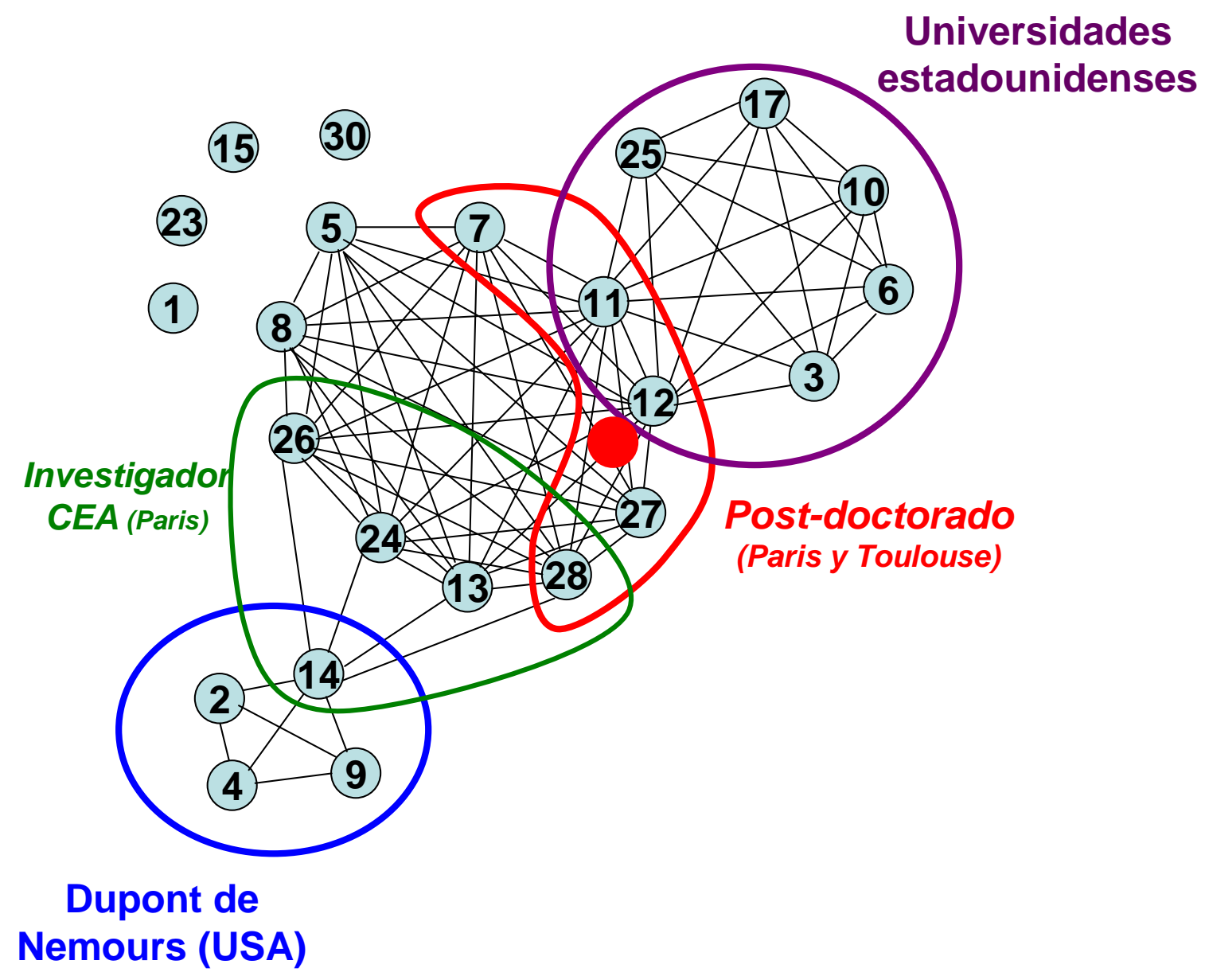

Figura 2. Calificación de la red de referencia del artículo publicado en PNAS en 2003

Las referencias que reenvían al investigador entrevistado están rodeadas en rojo. Entre ellas, las dos publicaciones del inicio ( $n-11$ y $n \circ 012$ ) son las que enlazan con las referencias de equipos estadounidenses, por medio de un investigador estadounidense con el que el equipo colaboró para estas dos publicaciones. La publicación aparecida en PNAS en 2003 fue el motivo de retomar relaciones con un investigador del equipo (CEA, París) con el que ellos ya habían colaborado en el 2000 y que forma el vínculo con un conjunto de publicaciones extraídas del mundo industrial. (Dupont de Nemours). De hecho, según el investigador entrevistado, el 
tema no habría continuado si este investigador no hubiera estado particularmente interesado por el proceso.

La forma de la red de referencia de la publicación muestra la relación de dos esferas en el equipo de París, la esfera académica y la esfera industrial. Es una relación que tuvo lugar por la instigación de un investigador del grupo y que se hace visible en la red de referencia de la publicación.

\section{Ejemplo de una red de referencia conexa}

La segunda publicación es un artículo largo de 8 páginas aparecido en 2004 en Inorganic Chemistry. Lo firmaron cinco autores, un estudiante de máster, un director de investigación del CNRS a su vez responsable de la publicación y a quien se entrevistó, un investigador y un técnico de su laboratorio, y un investigador de otro organismo de investigación parisino. 

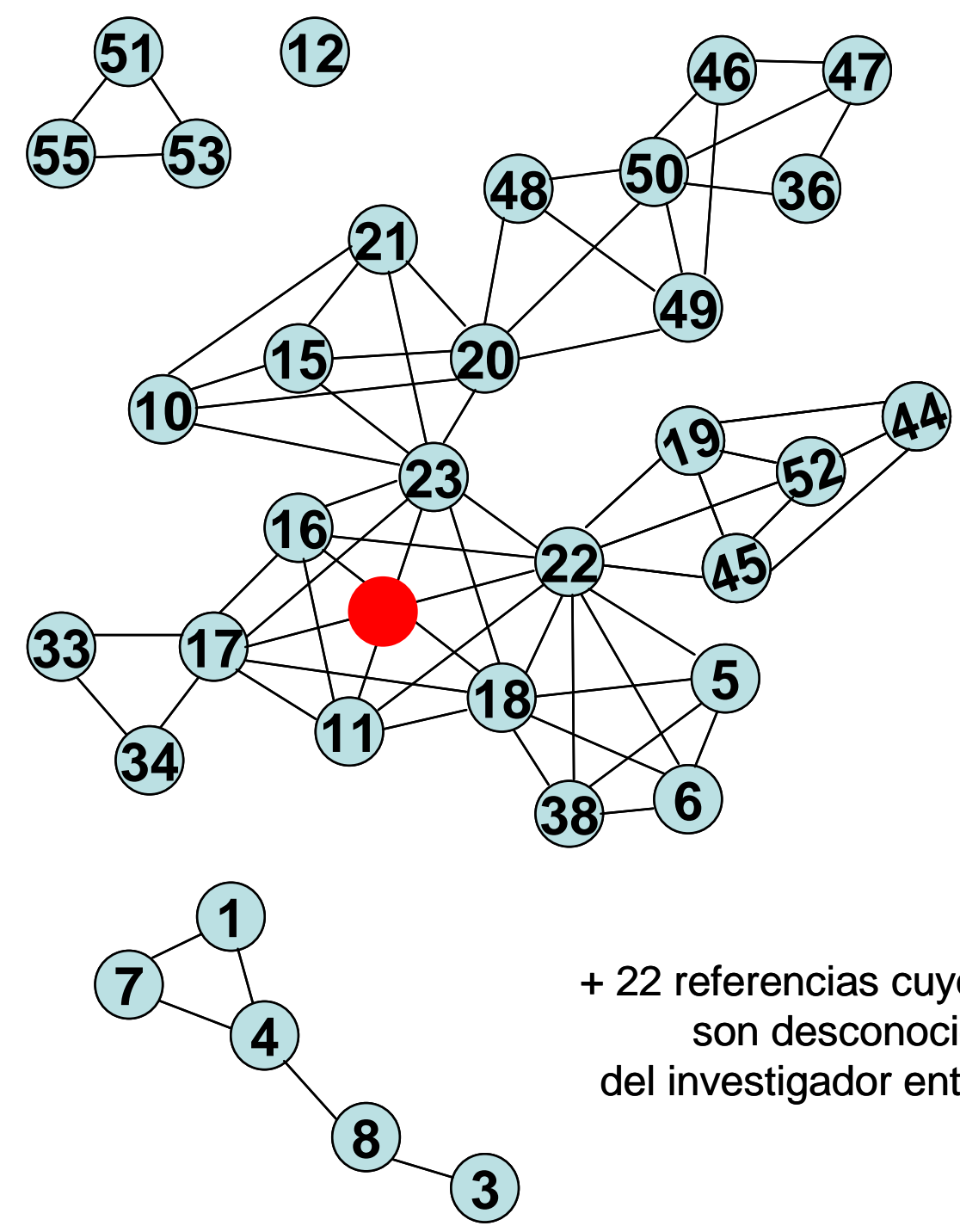

\section{+22 referencias cuyos autores son desconocidos del investigador entrevistado}

Figura 3. Red de referencia del artículo publicado en Inorganic Chemistry en 2004

La publicación incluye 35 referencias, el investigador entrevistado conoce al autor de al menos una de ellas, y a los autores de otras 22 referencias, no los conoce. Estas últimas son esencialmente referencias a medidas, programas o métodos de cálculo. Aunque menos polarizado que en el ejemplo anterior, la red de referencia también es bastante densa. Sólo encontramos una única referencia solitaria y dos componentes que no están relacionados con el resto del grupo. El investigador entrevistado está presente en las referencias $\mathrm{n} N 11,16,17,18,22$, у 23 (representado por el círculo rojo en las figuras 3 y 4 ) que es un componente más bien central alrededor del que se despliegan otros componentes directa 0 indirectamente adyacentes. ¿Por qué esta figura de referencias en la publicación? 


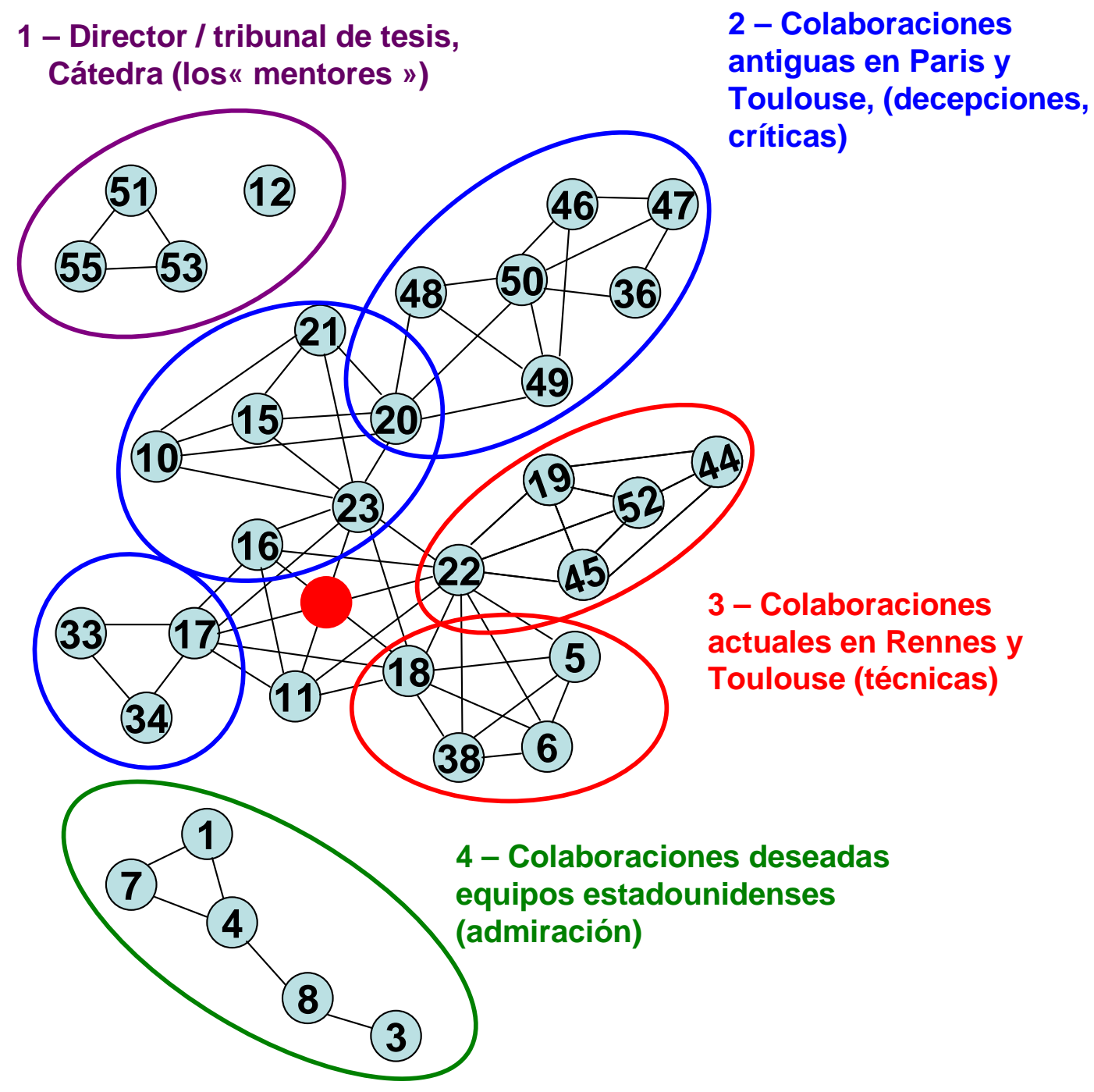

Figura 4. Calificación de la red de referencia del artículo publicado en Inorganic Chemistry en 2004

En realidad, la red de referencia marca el estado de las colaboraciones del autor que redactó el artículo solo, salvo la parte experimental. Volvemos a encontrar sus colaboraciones actuales (componentes " 3 ", marcados en rojo en la figura 4 anterior) útiles por razones técnicas (en física y química). También se citan colaboraciones más antiguas (componentes " 2 ", en azul en la figura 4) en comparación con las otras, de las que el autor expresa más bien decepciones criticando en varios aspectos a sus antiguos colaboradores. Los componentes no asociados ( 1 en violeta y 4 en verde en la figura 4) son personas por las que él dice profesar admiración (su antiguo director de tesis, un miembro del jurado de su titulación) y un equipo estadounidense con el que inició intercambios, precisamente al hilo de la redacción de este artículo.

La forma de la red de referencia de la publicación muestra a un investigador que multiplica a los colaboradores (lo que también se hace patente en su currículum) y, 
más concretamente, que está en fase de romper con antiguos colaboradores para iniciar otras colaboraciones, a su modo de ver, más prestigiosas.

\section{Ejemplo de una red de referencia dispersa}

La tercera publicación es un artículo corto de cuatro páginas firmado por cuatro autores: tres estudiantes de máster y un investigador al que se entrevistó. El artículo se publicó en 1999 en Angewandte Chemie, una revista muy prestigiosa. La publicación incluye 28 referencias, el investigador entrevistado conoce al menos a uno de los autores, y en 6 de las referencias, no ( $n^{\circ} 15,19,20,28,33$ y 34$)$.

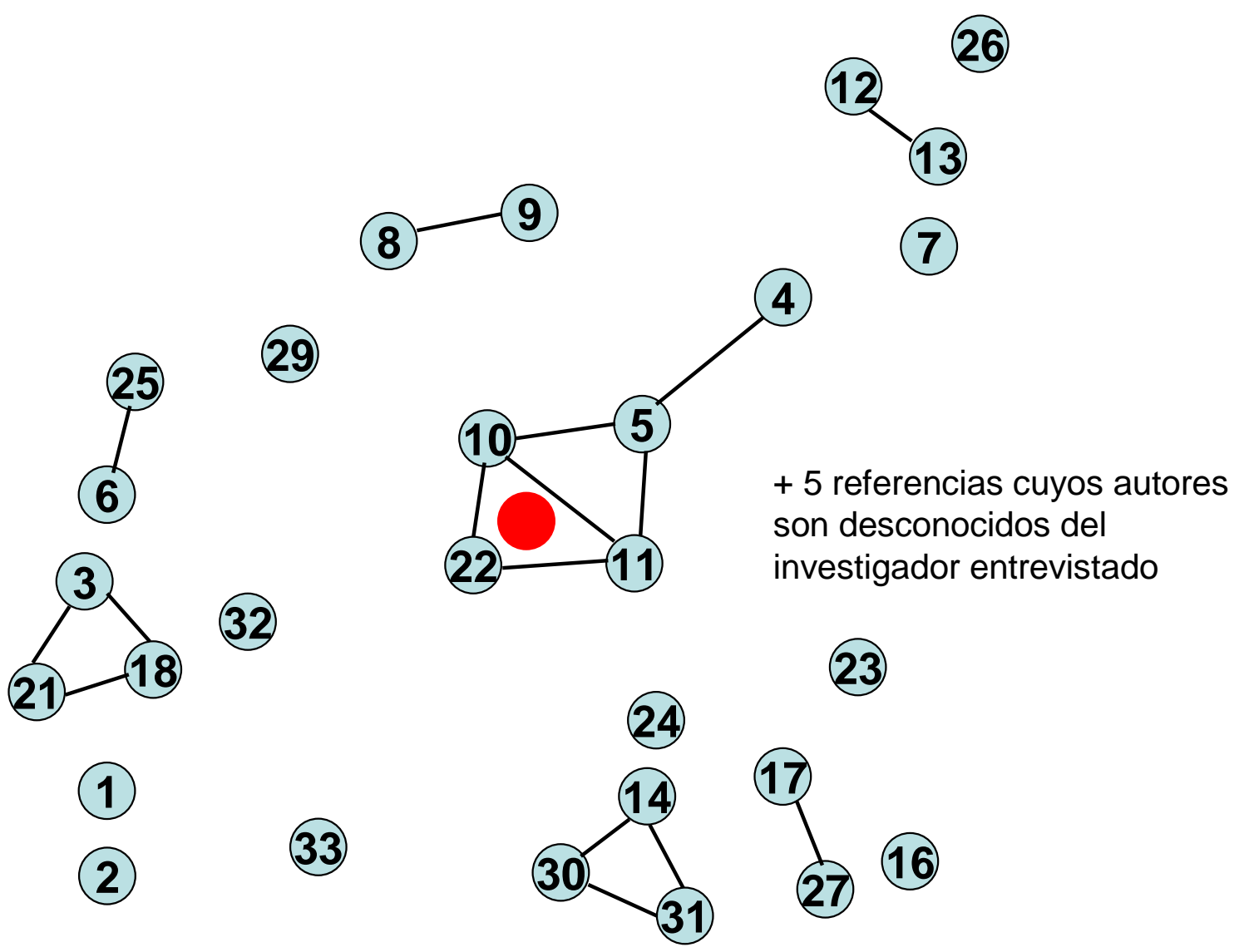

Figura 5. Red de referencia del artículo publicado en Angewandte Chemie en 1999

La red de referencia es muy poco densa. Excepto el componente donde encontramos las referencias del investigador entrevistado ( $n$ o 10, 11, 12, señaladas con el punto rojo en las figuras 5 y 6 ), sólo hay algunas tríadas y pares. ¿Cómo es posible que haya tal dispersión de las diferentes referencias de la publicación? El artículo comporta cierta profundidad histórica, ya que su objetivo es proponer una solución a un problema planteado en los años 20-30. Es también una especie de 
cierre de esta temática, ya que concluye una serie de publicaciones del equipo sobre el tema, y en especial las publicaciones citadas en este artículo, que datan de 1992, 1995, y 1997 (no 10, 11 y 12).

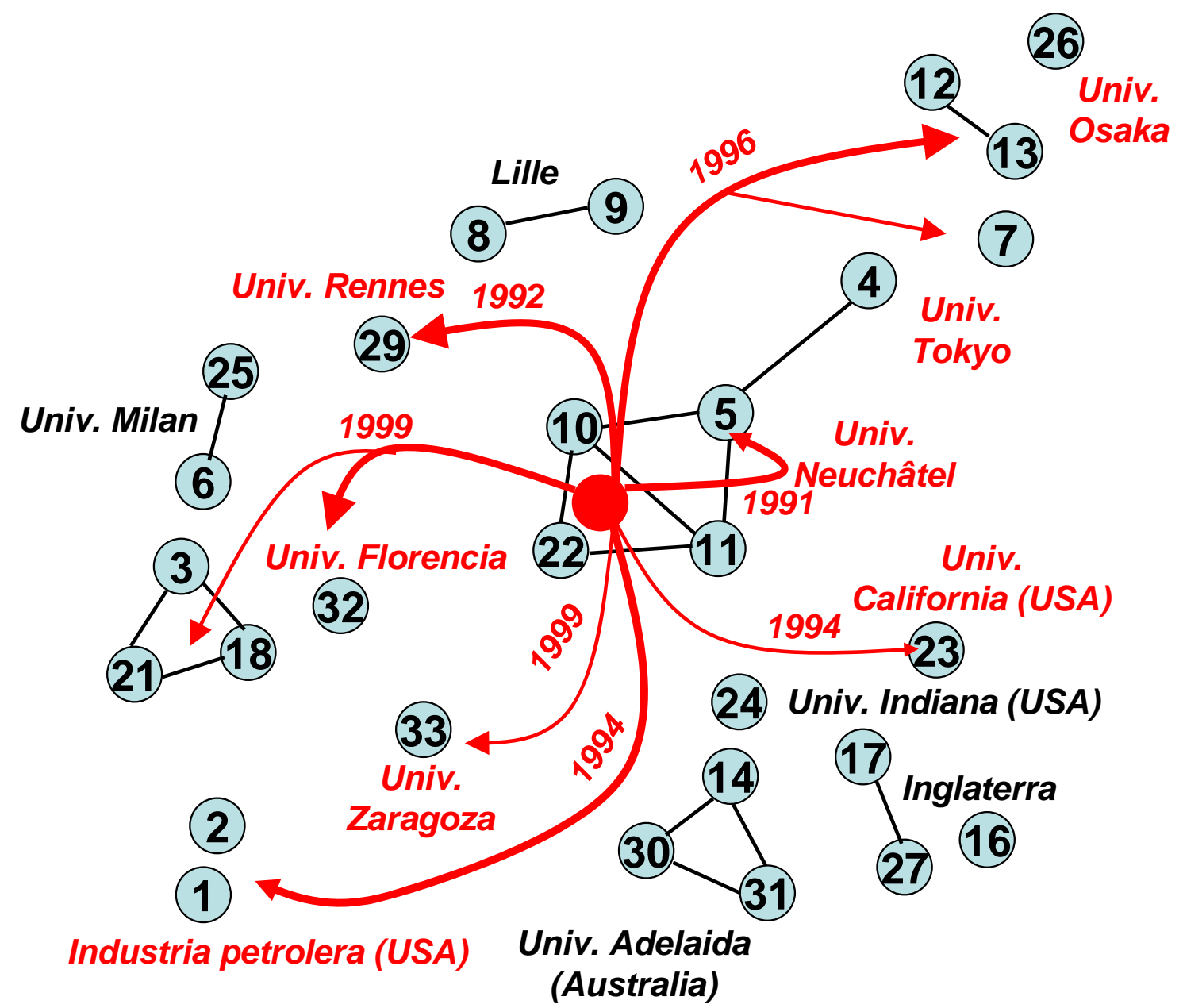

Figura 6. Calificación de la red de referencia del artículo publicado en Angewandte Chemie en 1999

La mayoría de las referencias reenvían a equipos universitarios del mundo entero que parecen haber trabajado independientemente en esta temática. Sin embargo, el investigador fue invitado a la mayoría de estos centros para dar conferencias estos últimos diez años (ver las fechas y las flechas rojas en la figura 6 anterior; un trazo más grueso señala una invitación de uno de los autores de la referencia) y él los conoce. Las referencias del artículo son "jalones" de sus diferentes viajes: "Sobre este tema, he hablado mucho, he hecho una gira por Alemania, una en Japón...". El carácter dispar de las referencias del artículo señala que este problema fue trabajado con frecuencia y que ya se hicieron preguntas al respecto.

En vista de la importancia del "descubrimiento", la publicación será clasificada como VIP (Very Important Paper)* por la revista y los trabajos del investigador serán

\footnotetext{
* Artículo muy importante
} 
ampliamente citados en un "high light" (artículo que destaca un tema en forma de resumen) el año siguiente a la publicación de este artículo. La dispersión de las referencias de esta publicación tal vez está relacionada con el hecho de que realmente ya no hay competencia en relación a los resultados que presenta.

Las redes de referencia como tales a menudo son el fruto y la expresión de una historia "personal", es decir, una historia cuyo autor principal del artículo es uno de los actores. El primer ejemplo ilustra una "reconexión" entre un equipo y un investigador que ya habían colaborado antes. Se manifiesta explícitamente un reencuentro entre ambos grupos, uno más bien académico y otro más bien industrial. El segundo artículo expresa más bien una voluntad, por parte del autor principal, de cambiar el orden de las cosas. Las relaciones expresadas son bastante subjetivas, y surgen sentimientos como la admiración, la crítica o la decepción. Los grupos se dibujan pues con bastante sutileza en un juego de proximidad y lejanía del autor con los demás. El tercer ejemplo corresponde al final de una temática que ocupó al autor durante varios años y que remata con este artículo de manera bastante gloriosa. Las relaciones entre los grupos están muy implícitas, como si las dinámicas estuvieran aún muy atenuadas. ¿Observamos en la literatura rastros de estos fenómenos?

\section{Evolución de los « universos de referencia » antes y después de la publicación}

Para observar la posible influencia que la publicación puede haber tenido en la literatura científica sobre los colectivos que ha puesto en escena, es necesario poder reunir los artículos anteriores o posteriores que comparten las mismas referencias que la publicación. Podemos considerar este corpus como representante del "universo de referencia" de las publicaciones estudiadas. En este corpus es donde hay que buscar rastros de un potencial impacto de dichas publicaciones.

Para cada artículo estudiado, hemos ${ }^{6}$ extraído de la Web of Science (WoS) la lista de las publicaciones que citan las mismas referencias que el artículo estudiado.

\footnotetext{
${ }^{6}$ En este trabajo, me ayudadó Mickael Soulier, estudiante en prácticas en el laboratorio de L3 (5o y 6a semestre) en el IUP (Instituto Universitario Profesional) SID (Estadística e Informática Decisional) en Toulouse, quien realizó pacientemente la extracción de miles de referencias. Gracias también a Yves Gingras por sus sugerencias en esta parte de la investigación.
} 


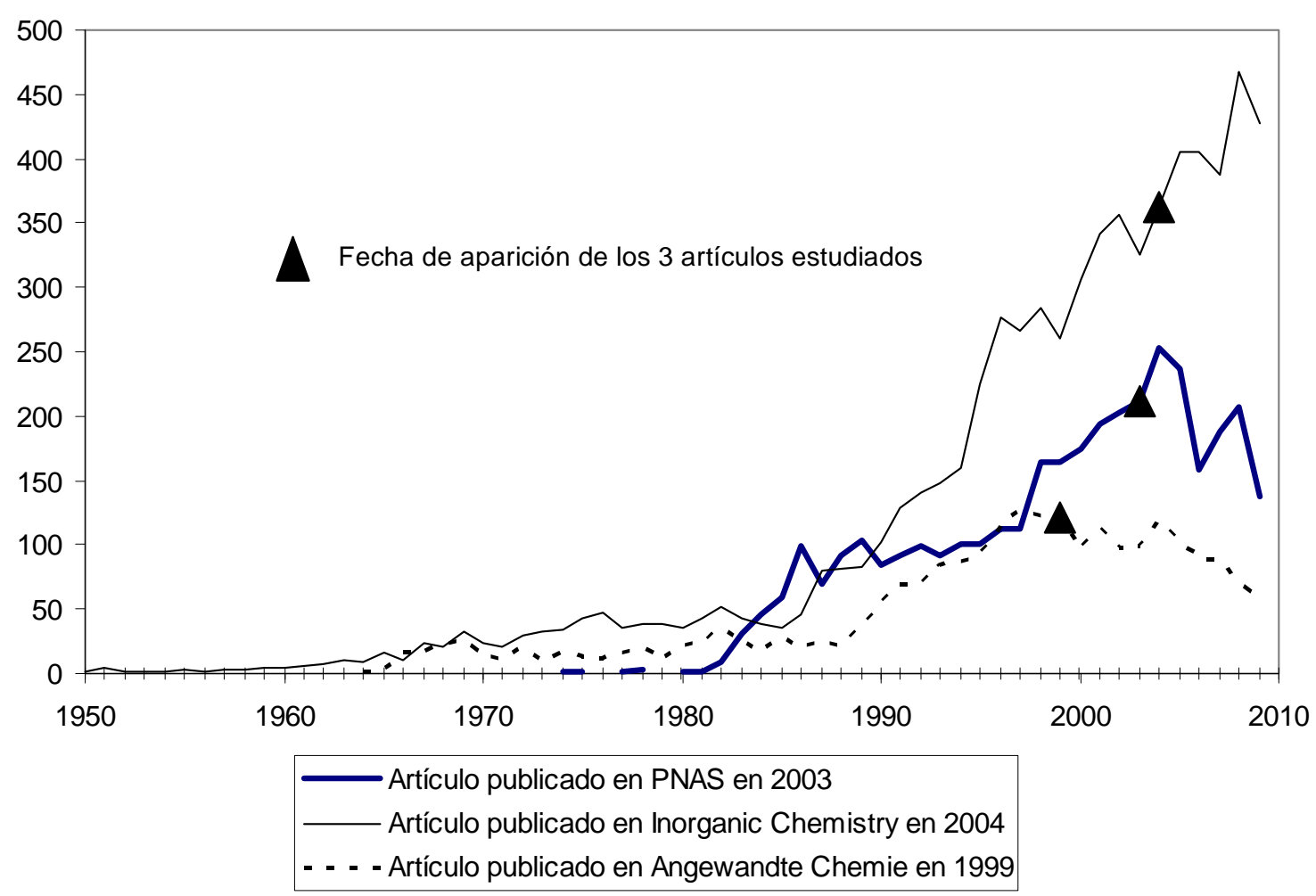

Figura 7. Número de publicaciones al año que citan al menos una misma referencia que el artículo estudiado

Como podemos ver en la figura 7 anterior, el universo de referencia del artículo publicado en PNAS está más bien en declive, el del artículo publicado en Inorganic Chemistry está en plena expansión, y el del artículo de Angewandte Chemie está más reducido que los otros y más bien estable.

Si nos interesamos en los colectivos científicos creados por referencias, es necesario poder identificar en la literatura las asociaciones entre las referencias. Por ello, en un segundo tiempo, hemos realizado redes de cocitacioes (Small, 1973), es decir, cartas relacionales de las referencias según estén cocitadas en un mismo artículo. ${ }^{7}$.

\footnotetext{
${ }^{7}$ Las redes de las figuras 7, 8 y 9 se diseñaron con el programa Netdraw.
} 


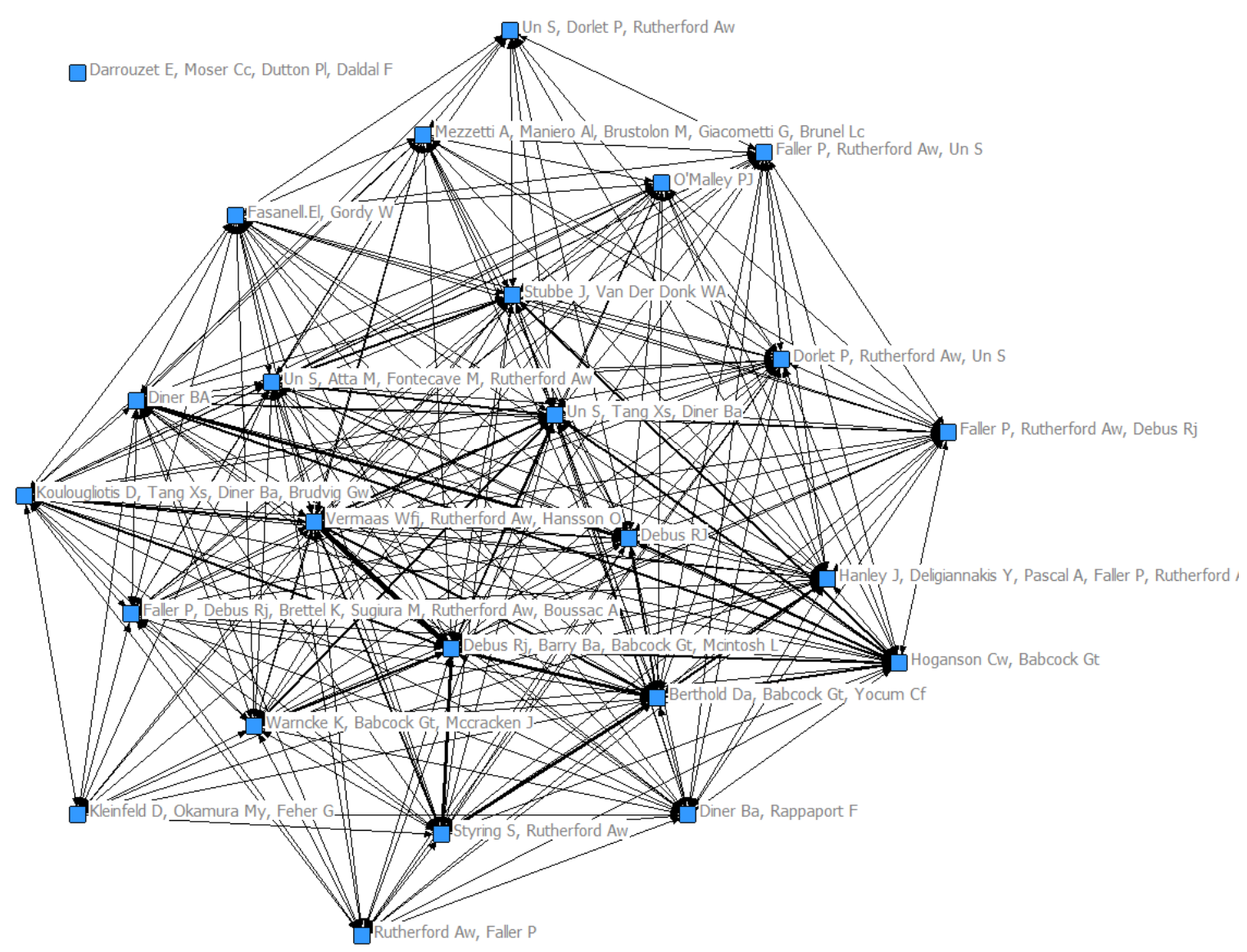

Figura 8. Red de cocitaciones de las referencias del artículo publicado en PNAS en 2003

Esta red (figura 8) representa los vínculos entre las referencias del artículo en función del número de publicaciones que las han cocitado en la literatura: Vermass et al. y Debus et al. en el centro de la red han sido cocitadas a menudo (187 veces), mientras que Un et al. y Mezzetti et al. (arriba) han sido cocitados en menos ocasiones (sólo 2 veces) y que Darrouzet et al. (arriba a la izquierda) nunca se asoció con ninguna otra referencia a parte del artículo estudiado.

Para comprender la medida en la que la publicación puede haber transformado este universo de referencia, es necesario estudiar la evolución de esta red. Por cada artículo estudiado, hemos contemplado dos periodos, tres años antes y tres años después. ¿Qué nos permite comprender la evolución de estas redes para cada artículo? 


\section{Estrechamiento de los vínculos entre dos colectivos en el universo de referencia}

En el primer artículo publicado en 2003 en Proceedings of The National Academy of Sciences, el reto para el equipo era proseguir una temática ya comprometida con otros dos artículos, en colaboración con un investigador universitario estadounidense. Esta prolongación se produjo tras la petición de otro investigador del equipo que no participó en los primeros trabajos, pero que estuvo muy interesado en el punto de vista teórico de estos resultados: "no habríamos continuado si S. no hubiera estado particularmente interesado por el procedimiento". Como vemos en la figura 2, la publicación fue lo que relacionó a los colaboradores del investigador universitario estadounidense ("grupo universidades estadounidenses") con los del colega investigador que salieron del grupo industrial Dupont de Nemours en EEUU, este último también estadounidense aunque con un puesto de investigador titular en Francia. ¿Podemos pues encontrar rastros de esta relación en la literatura científica?

\begin{tabular}{|c|c|c|c|c|c|}
\hline & & & & \\
\hline & & A & $\mathrm{B}$ & C & $\mathrm{T}$ \\
\hline \multirow{2}{*}{$\begin{array}{c}\text { Grupo "universidades } \\
\text { estadounidenses" (A) } \\
\text { - } 7 \text { referencias - }\end{array}$} & 2001-02-03 & $\begin{array}{c}76 \\
21 \%\end{array}$ & $\begin{array}{c}93 \\
26 \%\end{array}$ & $\begin{array}{r}195 \\
54 \%\end{array}$ & $\begin{array}{c}364 \\
100 \%\end{array}$ \\
\hline & 2004-05-06 & $\begin{array}{r}129 \\
28 \%\end{array}$ & $\begin{array}{l}202 \\
43 \% \\
\end{array}$ & $\begin{array}{r}138 \\
29 \%\end{array}$ & $\begin{array}{c}469 \\
100 \%\end{array}$ \\
\hline \multirow{2}{*}{$\begin{array}{l}\text { Grupo "Dupont de } \\
\text { Nemours" (B) } \\
-4 \text { referencias - }\end{array}$} & 2001-02-03 & $\begin{array}{c}93 \\
41 \% \\
\end{array}$ & $\begin{array}{l}16 \\
7 \% \\
\end{array}$ & $\begin{array}{r}120 \\
52 \% \\
\end{array}$ & $\begin{array}{c}229 \\
100 \% \\
\end{array}$ \\
\hline & 2004-05-06 & $\begin{array}{l}202 \\
65 \%\end{array}$ & $\begin{array}{l}25 \\
8 \%\end{array}$ & $\begin{array}{c}82 \\
27 \%\end{array}$ & $\begin{array}{c}309 \\
100 \%\end{array}$ \\
\hline \multirow{2}{*}{$\begin{array}{l}\text { Las demás }(\mathrm{C}) \\
\text { - } 14 \text { referencias - }\end{array}$} & 2001-02-03 & $\begin{array}{r}195 \\
45 \%\end{array}$ & $\begin{array}{l}120 \\
28 \%\end{array}$ & $\begin{array}{l}121 \\
28 \%\end{array}$ & $\begin{array}{c}436 \\
100 \%\end{array}$ \\
\hline & 2004-05-06 & $\begin{array}{r}176 \\
43 \% \\
\end{array}$ & $\begin{array}{r}113 \\
28 \% \\
\end{array}$ & $\begin{array}{r}117 \\
29 \% \\
\end{array}$ & $\begin{array}{c}406 \\
100 \% \\
\end{array}$ \\
\hline \multirow{2}{*}{ Total } & 2001-02-03 & $\begin{array}{r}364 \\
35 \% \\
\end{array}$ & $\begin{array}{r}229 \\
22 \% \\
\end{array}$ & $\begin{array}{l}436 \\
42 \%\end{array}$ & $\begin{array}{l}1029 \\
100 \%\end{array}$ \\
\hline & 2004-05-06 & $\begin{array}{l}507 \\
43 \% \\
\end{array}$ & $\begin{array}{l}340 \\
29 \%\end{array}$ & $\begin{array}{r}337 \\
28 \%\end{array}$ & $\begin{array}{r}1184 \\
100 \%\end{array}$ \\
\hline
\end{tabular}

Tabla 1. Evolución de la red de cocitaciones de las referencias del artículo publicado en PNAS en 2003

Entre los dos periodos, el número total de cocitaciones a penas aumenta ligeramente. No obstante, las referencias que reenvían a los grupos "universidades estadounidenses" ( $n$ - 3, 6, 10, 11, 12, 17 y 25 en la figura 2) y "Dupont de Nemours" ( $n^{\circ} 2,4,9,14$, idem) tuvieron tendencia a aumentar fuertemente. El grupo Dupont de Nemours está fuertemente asociado con el grupo de las universidades estadounidenses: el $65 \%$ de sus relaciones en lugar del $41 \%$ durante el periodo anterior. Asimismo, el grupo "universidades estadounidenses" 
estrechó sus relaciones con el grupo "Dupont de Nemours" (el 43\% contra el 26\% en el periodo anterior).

El objetivo del artículo, que consiste en relacionar dos grupos, es pues observable en la literatura, y el golpe de fuerza del artículo es quizá haber conseguido imponer el grupo "Dupont de Nemours" en la temática, o más exactamente haberlo anclado mejor en la temática asociándolo más sistemáticamente a un grupo ya instalado.

\section{Evolución de la centralidad de los grupos del universo de referencia}

El segundo artículo apareció en 2004 en Inorganic Chemistry. Es el resultado del trabajo de un investigador que trata de transformar su entorno profesional cambiando de colaboradores. El primero de los dos grupos con los que trabajó durante 15 años (entre otros) es parisino (ver figura 4, arriba en azul), el que inició la especialidad, por mucho tiempo "ineludible" en Francia. Sus críticas son bastante importantes: "Está decidido, ya no quiero volver a trabajar con él ni con ellos". El segundo grupo es estadounidense e incluye a un investigador conocido por el autor desde que hicieron su postdoctorado juntos en EEUU. Lo aprecia personalmente pero están en situación de competencia (publicaron un informe el mismo año sobre el mismo tema) y lamenta que a veces tenga que hacer co firmar a su ex "jefe" de postdoctorado para aumentar sus oportunidades de publicar. Según sus propias palabras se siente "un poco decepcionado" por él. Así mismo, aparecen en su artículo referencias a un grupo de investigadores europeos (belgas e ingleses) que por otro lado son personas a las que profesa respeto y con los que empezó a colaborar en la elaboración de este artículo (ver figura 4 anterior, componente en verde). De hecho, después de 2004, no colaborará más con los dos primeros grupos y proseguirá su colaboración con el tercero (tres publicaciones). ¿Vemos efectos de estos movimientos en el universo de referencia del artículo? 


\begin{tabular}{|c|c|c|c|c|c|c|}
\hline & & A & B & C & D & $\mathrm{T}$ \\
\hline \multirow{2}{*}{$\begin{array}{l}\text { Él y su equipo (A) } \\
-5 \text { referencias - }\end{array}$} & 2002-03-04 & & $\begin{array}{c}15 \\
22,7 \%\end{array}$ & $\begin{array}{c}44 \\
66,7 \%\end{array}$ & $\begin{array}{c}7 \\
10,6 \%\end{array}$ & $\begin{array}{c}66 \\
100,0 \%\end{array}$ \\
\hline & 2005-06-07 & & $\begin{array}{c}34 \\
27,6 \% \\
\end{array}$ & $\begin{array}{c}75 \\
61,0 \% \\
\end{array}$ & $\begin{array}{c}14 \\
11,4 \% \\
\end{array}$ & $\begin{array}{c}123 \\
100,0 \% \\
\end{array}$ \\
\hline \multirow{2}{*}{$\begin{array}{l}\text { Las colaboraciones } \\
\text { deseadas (B) } \\
-\quad 3 \text { referencias - }\end{array}$} & 2002-03-04 & $\begin{array}{c}15 \\
22,7 \% \\
\end{array}$ & & $\begin{array}{c}51 \\
77,3 \% \\
\end{array}$ & $\begin{array}{c}0 \\
0,0 \%\end{array}$ & $\begin{array}{c}66 \\
100,0 \% \\
\end{array}$ \\
\hline & 2005-06-07 & $\begin{array}{c}34 \\
43,0 \% \\
\end{array}$ & & $\begin{array}{c}34 \\
43,0 \% \\
\end{array}$ & $\begin{array}{c}11 \\
13,9 \% \\
\end{array}$ & $\begin{array}{c}79 \\
100,0 \%\end{array}$ \\
\hline \multirow{2}{*}{$\begin{array}{l}\text { Las colaboraciones } \\
\text { pasadas, un poco } \\
\text { decepcionantes (C) } \\
-\quad 4 \text { referencias - }\end{array}$} & 2002-03-04 & $\begin{array}{c}44 \\
36,7 \%\end{array}$ & $\begin{array}{c}51 \\
42,5 \%\end{array}$ & & $\begin{array}{c}25 \\
20,8 \%\end{array}$ & $\begin{array}{c}120 \\
100,0 \%\end{array}$ \\
\hline & 2005-06-07 & $\begin{array}{c}75 \\
56,8 \% \\
\end{array}$ & $\begin{array}{c}34 \\
25,8 \% \\
\end{array}$ & & $\begin{array}{c}23 \\
17,4 \% \\
\end{array}$ & $\begin{array}{c}132 \\
100,0 \%\end{array}$ \\
\hline \multirow{2}{*}{$\begin{array}{l}\text { Las colaboraciones } \\
\text { pasadas, criticadas (D) } \\
-\quad 4 \text { referencias - }\end{array}$} & 2002-03-04 & $\begin{array}{c}7 \\
21,9 \%\end{array}$ & $\begin{array}{c}0 \\
0,0 \%\end{array}$ & $\begin{array}{c}25 \\
78,1 \%\end{array}$ & & $\begin{array}{c}32 \\
100,0 \%\end{array}$ \\
\hline & 2005-06-07 & $\begin{array}{c}14 \\
29,2 \% \\
\end{array}$ & $\begin{array}{c}11 \\
22,9 \% \\
\end{array}$ & $\begin{array}{c}23 \\
47,9 \% \\
\end{array}$ & & $\begin{array}{c}48 \\
100,0 \% \\
\end{array}$ \\
\hline \multirow{2}{*}{ TOTAL } & 2002-03-04 & $\begin{array}{c}66 \\
23,2 \% \\
\end{array}$ & $\begin{array}{c}66 \\
23,2 \% \\
\end{array}$ & $\begin{array}{c}120 \\
42,3 \% \\
\end{array}$ & $\begin{array}{c}32 \\
11,3 \% \\
\end{array}$ & $\begin{array}{c}284 \\
100,0 \% \\
\end{array}$ \\
\hline & 2005-06-07 & $\begin{array}{c}123 \\
32,2 \% \\
\end{array}$ & $\begin{array}{c}79 \\
20,7 \% \\
\end{array}$ & $\begin{array}{c}132 \\
34,6 \% \\
\end{array}$ & $\begin{array}{c}48 \\
12,6 \%\end{array}$ & $\begin{array}{c}382 \\
100,0 \% \\
\end{array}$ \\
\hline
\end{tabular}

Tabla 2. Evolución de la red de cocitaciones de las principales referencias del artículo publicado en Inorganic Chemistry en 2004

La red de cocitaciones entre grupos aumentó fuertemente entre los dos periodos, así como aumentó el número total de citaciones a las referencias del artículo (ver la curva en la figura 8 anterior). El grupo que se benefició más de este incremento de la conexión entre los grupos es el del autor del artículo (él, sus estudiantes y sus colaboradores "técnicos" regulares). El que perdió más conexiones con los otros es el que tenía más, el grupo de su ex colega de postdoctorado, convertido en su principal competencia. Los otros dos grupos tienen un peso constante. Más allá del número de vínculos, podemos darnos cuenta, entre los dos periodos, de una centralidad acrecentada del equipo del investigador entrevistado: están menos asociados exclusivamente al grupo de su ex colega y competencia (grupo C), y se asocian con más frecuencia a los demás, y en especial al grupo del que el investigador entrevistado esperaba mucho en cuanto a colaboraciones en 2005 (grupo B). El perfil de vínculos de los otros grupos muestra que en lo sucesivo el investigador y su equipo están asociados muy a menudo a ellos: más de la mitad de los vínculos del equipo de su competidor y cerca de la mitad de los que él espera colaboración. El resultado del artículo consiste pues en volver a centrar el universo de referencia sobre el autor, convertido en una referencia mucho más ineludible. 


\section{El fin de una temática o la desaparición de un universo de referencia}

El tercer artículo apareció en una revista muy prestigiosa y generalista, Antgewante Chemie, una revista alemana que también publica una edición internacional. Es un artículo particular ya que es el último de una serie, y da fin a la temática para el autor, que pasó a otra después. De hecho, no se manifiesta especialmente respecto a esta publicación. El artículo dio por concluido sus trabajos, comenzados algunos años atrás: "Esta es una referencia a nosotros. Entramos en esta temática con este artículo. De todas formas respeto las demás, porque la doy en sexta posición". El universo de referencia del artículo es más reducido que los otros artículos, en especial que el anterior, y tuvo tendencia a disminuir desde hace algunos años: el número global de citaciones en las referencias del artículo pasó de 370 entre 1997 y 1999 a 306 artículos entre 2000 y 2002 (ver figura 8 anterior). La evolución de la red de cocitaciones a las referencias del artículo muestra una evolución bastante radical después de 1999 .

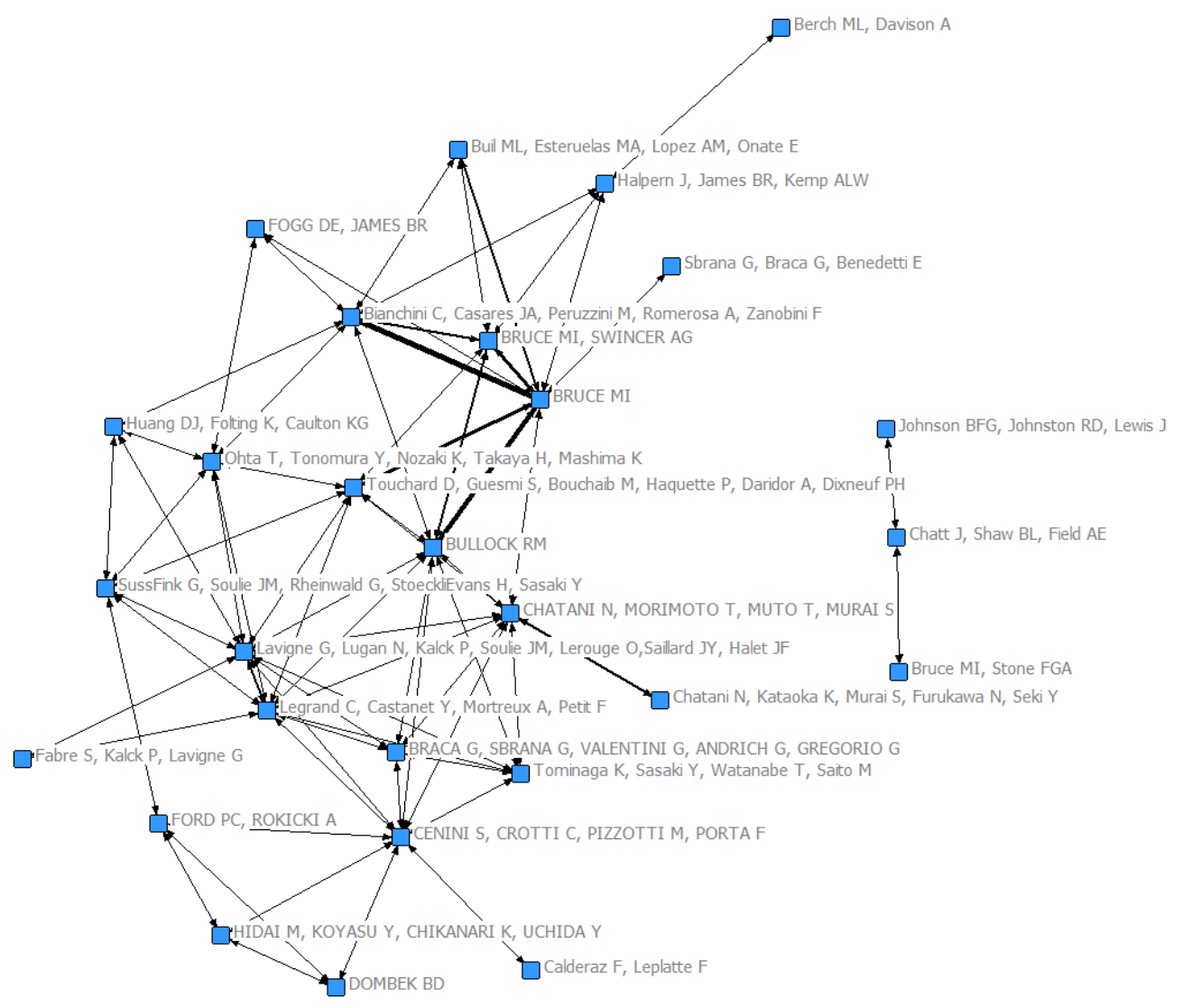

Figura 9. Red de cocitaciones (de 1997 a 1999) de las referencias del artículo publicado en Angewandte Chemie en 1999 


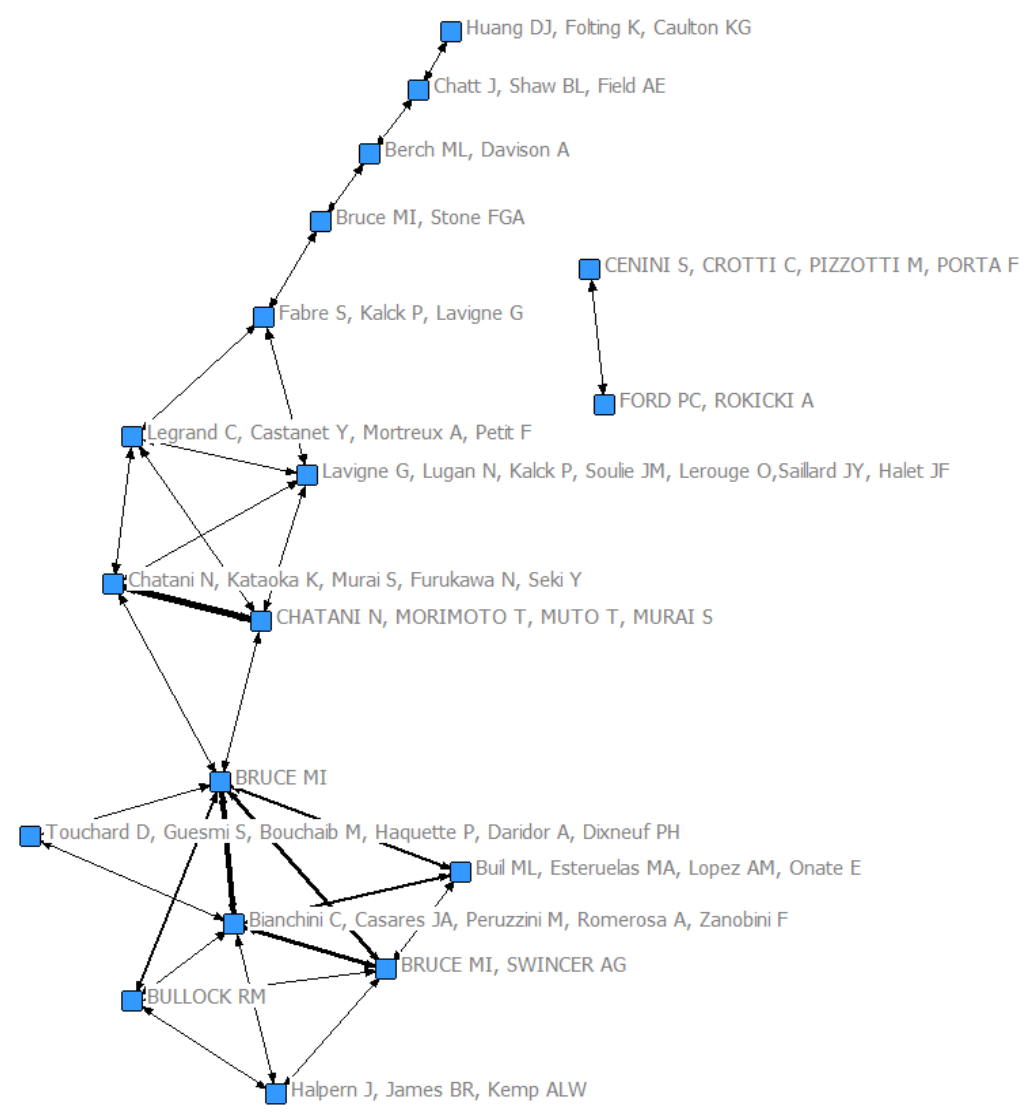

Figura 10. Red de cocitaciones (de 2000 a 2002) de las referencias del artículo publicado en Angewandte Chemie en 1999

Entre los dos periodos, el número de referencias implicadas en la red de cocitaciones disminuyó, pasando de 28 a 18. El número de cocitaciones no disminuyó excesivamente pero la conexión entre las referencias 0 , dicho de otra forma, la densidad de la red, pasó de 70 a 29. Esto significa que las cocitaciones tuvieron tendencia a centrarse sobre algunas referencias. Efectivamente, la red de las cocitaciones era bastante difusa y con forma de estrella en el primer periodo, y se apoyó y volvió a centrarse en algunas referencias en el segundo. Aquí tenemos la ilustración del fin de una temática que, dicho por el propio autor en la entrevista, se consiguió en gran parte gracias a su artículo (clasificado por la revista como uno de los dos Very Important Paper* del año). Se cerró también a razón del “High Light" que se publicó en el 2000 y que hacía una síntesis sobre esta temática, citando tres de las publicaciones del autor entrevistado y trabajando sobre una investigación futura. En definitiva, casi podríamos decir que el éxito alcanzado por esta publicación fue la señal manifiesta de la desaparición del universo de referencia.

\footnotetext{
* Artículo muy importante
} 


\section{Conclusión}

En este texto he tratado de mostrar que la red de referencia es una especie de estado (de fotografía) de las relaciones del investigador, para el que hay un "antes" y un "después". Esta dinámica se hace visible con el estudio del universo de referencia del artículo, es decir, la configuración de referencias tal y como son citadas y cocitadas en la literatura.

Los tres ejemplos estudiados presentan universos de referencia con dinámicas diferentes. Mientras el primero presenta una configuración enfocada a reforzar la conexión entre dos colectivos, el segundo marca la trasformación de los equilibrios entre cuatro equipos especializados en el mismo objeto. La dinámica del tercer texto es también diferente porque revela una decadencia rápida de los vínculos en cuanto se publica el artículo. Incluso a través de estos únicos tres ejemplos, podemos asumir que el "universo de referencia" de un artículo no siempre está en expansión (ejemplo 3), que no siempre gira entorno al que lo ha construido (ejemplo 1), y que puede esconder tensiones fuertes (ejemplo 2). Sería pues particularmente interesante continuar estudiando casos.

Mi propósito no es decir que el texto estudiado produjo directamente las transformaciones del universo de referencia que describí antes; también es el resultado, ya que el autor elaboró su texto y su bibliografía en base a este universo de referencia. Sin embargo, el análisis presentado aquí, dado que parte del artículo y del punto de vista de su autor, permite comprender mejor lo que puede ser la dinámica de un texto científico a nivel relacional y corolariamente, cognitivo. A este nivel, hay que subrayar la importancia de las informaciones cualitativas derivadas de entrevistas realizadas a los investigadores. Son ciertamente indispensables, no solamente para comprender las dinámicas, sino también para saber dónde encontrarlas. 


\section{Bibliografía}

Baldi, Stephane (1998). "Normative versus Social Constructivist Processes in the Allocation of Citations: A Network-Analytic Model". American Sociological Review, vol. 63, n6, pp. 829-846.

Brooks, Terrence A. (1985). "Private Acts and Public Objects: An Investigation of Citer Motivations". J ournal of the American Society for Information Science, vol. 36, $n^{\circ} 4$, pp. 223-229.

Brooks, Terrence A. (1986). "Evidence of Complex Citer Motivations". J ournal of the American Society for Information Science, vol. 37, $n^{\circ} 1$, pp. 34-36.

Callon, Michel, Françoise Bastide, Serge Bauin, Jean-Pierre Courtial et William Turner (1984). "Les mécanismes d'intéressement dans les textes scientifiques". Cahiers STS, $n^{\circ} 4$, pp. 88-105.

Callon, Michel, Jean-Pierre Courtial et Hervé Penan (1993). La scientométrie, Paris: PUF, "Que sais-je ?".

Callon, Michel, John Law et Arie Rip (1986). Mapping the dynamics of science and technology. Londres: The MacMillan Press Ltd.

Chubin, Daryl E. et Soumyo D. Moitra (1975). "Content Analysis of References: Adjunct or Alternative to Citation Counting?" Social Studies of Science, vol. $5, n^{\circ} 4$, pp. 423-441.

Cole, Stephen et Jonathan R. Cole (1967). "Scientific Output and Recognition: A Study in the Operation of the Reward System in Science". American Sociological Review, vol. 32, n³, pp. 377-390.

Crane, Diana (1972). Invisible Colleges. Diffusion of knowledge in scientific communities. Chicago: The University of Chicago Press.

Cronin, Blaise (1984). The Citation Process: The Role and Significance of Citations in Scientific Communication. London: Taylor Graham.

Cronin, Blaise et Debora Shaw (2002). "Identity-creators and image-makers: Using citation analysis and thick description to put authors in their place". Scientometrics, vol. $54, n^{\circ} 1$, pp. 31-49.

Gilbert, Nigel (1977). "Referencing as persuasion". Social Studies of Science, vol. 7, $\mathrm{n}^{\circ} 1$, pp. 113-122. 
Grossetti, Michel et Béatrice Milard (2003). “Les évolutions du champ scientifique en France à travers les publications et les contrats de recherche". Actes de la Recherche en Sciences Sociales, $n^{\circ} 148, p p .47-56$.

Hargens, Lowell L. (2000). “Using the Literature: Reference Networks, Reference Contexts, and the Social Structure of Scholarship". American Sociological Review 65, no. 6 (Décembre): 846-865.

Hargens, Lowell L. et Diane H. Felmlee (1984). "Structural determinants of stratification in science". American sociological review, vol. 49, n5, pp. 685-697.

Harwood, Nigel (2008). "Publication outlets and their effect on academic writers' citations". Scientometrics, vol. 77, n², pp. 253-265.

Latour, Bruno (1977). “La rhétorique de la science. «Pouvoir » et « devoir » dans un article de science exacte". Actes de la recherche en sciences sociales, $n^{\circ} 13, p p$. 81-95.

MacRoberts, Michael H. et Barbara R. MacRoberts (1984). "The Negational Reference: Or the Art of Dissembling". Social Studies of Science, Vol.14, $n^{\circ} 1, p p$. 91-94.

Merton, Robert K. (1968). "The Matthew Effect in Science: The reward and communication systems of science are considered". Science, vol. 159, n³810, pp. 56-63.

Michaelson, Alaina G. (1993). "The development of a scientific specialty as diffusion through social relations: the case of role analysis". Social Networks, vol. $15, \mathrm{n}^{\circ} 3$, pp. 217-236.

Milard, Béatrice (2010). “Quelles sociabilités derrière les références bibliographiques? L'exemple d'articles de chimie". Communication aux 2ème journées d'études « Réseaux sociaux », Toulouse, 16-17 mars 2010.

Moravcsik, Michael J. et Poovanalingam Murugesan (1975). "Some Results on the Function and Quality of Citations". Social Studies of Science, vol. 5, no. 1, pp. 8692.

Mullins, Nicolas C. (1972). "The development of a scientific specialty: the phage group and the origine of molecular biology". Minerva, vol. 10, ${ }^{\circ} 1$, pp. 51-82.

Price, Derek J. De Solla (1965). "Networks of Scientific Papers". Science, vol. 149, $n^{\circ} 3683$, pp. 510-515.

Price, Derek J. De Solla (1966). "Collaboration in an invisible college". American psychologist, $n^{\circ} \mathrm{XXI}$, pp. 1011-18. 
Price, Derek J. de Solla (1970). "Citation measures of hard science, soft science, technology, and nonscience" in C. E. Nelson and D. K. Pollack (eds.), Communication among Scientists and Engineers. Lexington, MA: Heath, pp. 3-22.

Roth, Camille (2008). “Coévolution des auteurs et des concepts dans les réseaux épistémiques : le cas de la communauté "zebrafish »". Revue Française de Sociologie, vol. $49, n^{\circ} 3$, pp.523-558.

Small, Henry et Belver C. Griffith (1974). "The Structure of Scientific Literatures I: Identifying and Graphing Specialties". Science Studies, vol.4, n¹, pp. 17-40.

Small, Henry (1979). "Specialties and disciplines in sciences and social sciences: an examination of their structure using citation indexes". Scientometrics, vol. $1, n^{\circ} 5-6$, pp. 445-461.

Tuire, Palonen et Lehtinen Erno (2001). “Exploring invisible scientific communities: Studying networking relations within an educational research community. A Finnish case". Higher Education, vol. 42, n4, pp. 493-513.

White, Howard D. (2001). "Authors as Citers over Time". Journal of the American Society for Information Science \& Technology, vol. 52, $n^{\circ} 2$, pp. 87-108.

White, Howard D., Barry Wellman et Nancy Nazer (2004). "Does citation reflect social structure?: longitudinal evidence from the "Globenet" interdisciplinary research group". Journal of the American Society for Information Science \& Technology vol. 55, $n^{\circ} 2$, pp. 111-126. 